\title{
Family Physicians with a Sports Medicine Certificate of Added Qualification (CAQ): Well Prepared to Meet a Significant Patient Care Need
}

\author{
John P. DiFiori, MD, and James Kinderknecht, MD
}

\section{Keywords: Sports Medicine}

In this issue, Rankin, Cochrane, and Puffer report on the practice patterns of family physicians with Certificates of Added Qualifications (CAQs) in Sports Medicine. ${ }^{1}$ Of the 87,610 board-certified family physicians in the United States, 2.4\% ( $\mathrm{n}=$ 2061) currently hold a sports medicine CAQ (James Puffer, MD, Executive Director, ABFM, personal communication). As mentioned in their article, despite the 23 years that the CAQ has been offered, there is little information on the relative amount of time that the family physicians who are certified in sports medicine spend in the practice of family medicine.

Using the response to a single question posed to those applying to sit for the CAQ recertification examination between 2005 and 2013, Rankin et $\mathrm{al}^{1}$ report that for the 1875 physicians who applied during this time, the majority spent more than 50\% of their time practicing sports medicine. These findings are similar to those reported by Peterson et $\mathrm{al}^{2}$ using a similar methodology for family physicians with a CAQ in Geriatrics. In addition, Rankin et al report a significant trend toward an

From the Division of Sports Medicine and Non-Operative Orthopaedics, University of California, Los Angeles (JPD); Hospital for Special Surgery, New York (JK); Weill Cornell Medical College, New York (JK).

Funding: none.

Conflict of interest: none declared.

Corresponding author: John P. DiFiori, MD, UCLA Department of Family Medicine, Division of Sports Medicine and Non-Operative Orthopaedics, 10833 Le Conte Ave. 50-080 CHS, Los Angeles, CA 90095 (E-mail: JDiFiori@mednet.ucla.edu).

\section{See Related Article on Page 695.}

increase in the relative amount of time spent practicing sports medicine over this survey period.

Membership data from the American Medical Society for Sports Medicine (AMSSM) is generally consistent with the findings of Rankin et al. ${ }^{1}$ The AMSSM, with over 2700 members, is the largest organization of sports medicine physicians in the United States. Family physicians make up $73 \%$ of the membership. ${ }^{3}$ More than $90 \%$ hold a CAQ in sports medicine or are eligible to receive a CAQ. The 2014-2015 membership survey found that $64 \%$ of members (including those other than family physicians) spend at least $50 \%$ of their time practicing sports medicine; $20 \%$ reported practicing exclusively sports medicine.

Additional information comes from the 2015 AMSSM membership practice survey. Of the 594 respondents, $70 \%$ were family physicians. ${ }^{4}$ Approximately $7 \%$ reported practicing only in an orthopedic clinic, with another $14 \%$ practicing entirely in a combination of an orthopedic clinic and a sports medicine clinic.

Thus, the findings of 2 AMSSM reports, using somewhat different questions, are consistent in that approximately $20 \%$ of sports medicine physicians do not practice their primary specialty. While supporting the findings of Rankin et al, ${ }^{1}$ it is clear the respondents of these surveys are largely the same family physician population, that is, these were essentially 3 surveys of the same physicians.

One likely reason for the findings reported in these surveys is the substantial need for physicians trained in sports medicine. It is well established that orthopedic conditions are among the most frequent reasons for referral in family medicine offices. ${ }^{5}$ Furthermore, the rate of orthopedic referral is increasing significantly. ${ }^{6}$ Yet only a very small 
percentage of these cases ultimately require surgery. ${ }^{7}$ Thus, given this growing demand, it is noteworthy that approximately $80 \%$ of family physicians with a sports medicine CAQ continue to practice some amount of family medicine.

Another issue driving the findings of Rankin et $\mathrm{al}^{1}$ may be compensation. Although compensation data based on the relative percentage of time spent practicing family medicine versus sports medicine is not directly available, the practice of musculoskeletal medicine lends itself to greater opportunities to bill for new patients, consultations, and procedures, which may increase compensation.

Other factors that are not measured in the available data could also play a role. For example, do younger family medicine physicians with a sports medicine CAQ spend a greater proportion of their time practicing family medicine, and "specialize" more as their practice matures? Or are younger sports medicine physicians more attracted to exclusively musculoskeletal practices, perhaps because of better compensation packages? Do practice patterns vary by region of the country? How is time devoted to serving as a team physician assessed and interpreted? Since this is typically time spent outside of the office in an athletic training facility and/or providing on-site event coverage, the current data may actually underestimate the "sports medicine" time component.

The available data indicate that approximately $20 \%$ of family physicians with a sports medicine CAQ do not practice any family medicine. However, it is important to recognize that the practice of "sports medicine" is not necessarily the same as "musculoskeletal medicine." The practice of sports medicine encompasses the diagnosis and treatment of a multitude of nonmusculoskeletal conditions such as infectious diseases, psychiatric issues, sports-related concussions, and cardiac conditions-all of which are encountered in a family medicine practice. This is especially true when functioning in the role of a team physician. In many such circumstances, the team physician serves as the primary care physician for a population of athletes, providing care for both musculoskeletal problems as well the gamut of primary care concerns.
Thus, in this role, these family physicians may view themselves as "family physicians practicing sports medicine" rather than simply "sports medicine physicians."

Future surveys need to address the limitations of the existing data. For now, it is important to appreciate that family physicians already receive significantly more required residency training in musculoskeletal conditions, and sports medicine specifically, than other primary care specialties. Those who choose to pursue fellowship training in sports medicine, and ultimately a CAQ, further expand on their family medicine training and gain a high level of proficiency in the broad skills needed to serve as sports medicine physicians. It would seem that the specialty is providing a much needed level of secondary care for which the demand is increasing. The fact that family physicians are well prepared to meet this demand benefits patients and reflects well on the specialty.

\section{References}

1. Rankin WM, Cochrane A, Puffer JC. Family physicians with a certificate of added qualifications in sports medicine spend the majority of their time practicing sports medicine. J Am Board Fam Med 2015;28:695-6.

2. Peterson LE, Cochrane A, Bazemore AW, Petterson S. Reported practice patterns among family physicians with a geriatrics certificate of added qualifications. J Am Board Fam Med 2015;28:314-5.

3. American Medical Society for Sports Medicine, American Medical Society for Sports Medicine Foundation. 2014-2015Annual report. Available from: https:// www.amssm.org/Content/pdf\%20files/2015_AnnualRpt. pdf. Accessed September 26, 2015.

4. American Medical Society for Sports Medicine. Recent graduate practice and salary survey 2015. available from: http://www.amssm.org/Fellowships.html.

5. Forrest CB, Nutting PA, Starfield B, von Schrader S. Family physicians' referral decisions: results from the ASPN referral study. J Fam Pract 2002;51:215-22.

6. Barnett ML, Song Z, Landon BE. Trends in physician referrals in the United States, 1999-2009. Arch Intern Med 2012;172:163-70.

7. Menzies RD, Young RA. Referrals from a primary care-based sports medicine department to an orthopaedic department: a retrospective cohort study. Br J Sports Med 2011;45:1064-7. 